\title{
ORTHOSIMILAR EXPANSION SYSTEMS IN SPACE WITH REPRODUCING KERNEL
}

\author{
V.V. NAPALKOV (JR.)
}

\begin{abstract}
We study expansion system similar to orthogonal ones (orthosimilar systems) in reproducing kernel Hilbert spaces. We establish the equivalency of two definitions of orthosimilar system. We show the relation of orthosimilar system with the problem on description of the dual space to a Hilbert space in terms of a special system of functions.
\end{abstract}

Keywords: Bergman space, Hilbert spaces, reproducing kernel, reproducing kernel Hilbert space, Paley-Wiener theorem.

Mathematics Subject Classification: 30H20, 30E10, 30E20, 32A26, 46E22, 47B32

Expansions systems similar to orthogonal ones (orthosimilar expansion systems) in a Hilbert space were introduced by T.P. Lukashenko in work [1] and they are applied, say, in wavelet analysis. In this work we study orthosimilar expansion systems in spaces with reproducing kernel. The need of studying spaces with reproducing kernel is motivated by the problems of complex analysis.

Definition 1 (See, for instance, [3]). Let $H$ be a Hilbert space over field $\mathbb{C}$ consisting of functions defined on a set $M$. Space $H$ is called reproducing kernel Hilbert space if for each point $z_{0} \in M$ the functional

$$
\delta_{z_{0}}: H \longrightarrow \mathbb{C} ; \quad \delta_{z_{0}} f \longrightarrow f\left(z_{0}\right), f \in H
$$

is linear and continuous on $H$.

By Riesz-Fischer theorem, a linear continuous functional on Hilbert space $H$ is generated by some element of $H$. The identity

$$
f(\xi)=\delta_{\xi} f=\left(f(z), K_{H}(z, \xi)\right)_{H}, \quad \xi \in M,
$$

determines the reproducing kernel of space $H$ as a function $K_{H}(z, \xi)$ of two variables $z, \xi \in M$. The main properties of Hilbert spaces with reproducing kernel are presented, for instance, in [3]. The most important fact of the theory of spaces with reproducing kernel is the following Moore-Aronszajn theorem (see, for instance, [5]).

Remark. We assume for simplicity that $H$ is a Hilbert space over the field of complex numbers. For Hilbert spaces over the field of real numbers all said below is also true with appropriate changes.

Theorem A. Let $M$ be an arbitrary set of points, and $K(z, \xi): M \times M \rightarrow \mathbb{C}$ is a complexvalued function. This function is a reproducing kernel of some Hilbert space $H$ consisting of complex-valued functions defined on $M$ if and only if for each finite set of points $z_{1}, z_{2}, \ldots, z_{n} \in$ $M$ and for each finite set of complex numbers $c_{1}, c_{2}, \ldots, c_{n}$ the condition

$$
\sum_{l, m=1}^{n} c_{l} \cdot \overline{c_{m}} \cdot K\left(z_{l}, z_{m}\right) \geqslant 0
$$

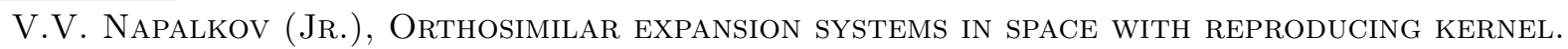

(c) NAPALKOV V.V. (JR.) 2013.

Submitted June 19, 2013. 
holds true. At that, $H$ is the only space with reproducing kernel having function $K(z, \xi)$ as the reproducing kernel.

In works of T.P. Lukashenko [1], [2] the following definition of orthosimilar expansion system is given.

Remark. The definition of orthosimilar expansions system the notion of Lebesgue integral with the values in a Hilbert space is used. The theory of such integrals is exposed in [4]. To distinguish the case when the integral is treated as the usual Lebesgue integral, we introduce the notation: the symbol $\int_{\Omega}^{(H)}$ indicates the integral of a function with values in a Hilbert space $H$ (see below).

Definition 2. (see [1]) Let $H$ be a Hilbert space over field $\mathbb{R}$ or $\mathbb{C}$, and $\Omega$ is the space with a countable additive measure $\mu$. A system of elements $\left\{e_{\omega}\right\}_{\omega \in \Omega}$ is called orthosimilar (similar to orthogonal) expansion system with the measure $\mu$ in $H$, if each element $y \in H$ can be represented as

$$
y=\int_{\Omega}^{(H)}\left(y, e_{\omega}\right)_{H} e_{\omega} d \mu(\omega)
$$

where the integral is treated as the proper or improper Lebesgue integral. In the latter case there exists an exhaustion $\left\{\Omega_{k}\right\}_{k=1}^{\infty}$ of space $\Omega$, namely, all $\Omega_{k}$ are $\mu$-measurable, $\Omega_{k} \subset \Omega_{k+1}$ as $k \in \mathbb{N}$ and $\bigcup_{k=1}^{\infty} \Omega_{K}=\Omega$. This exhaustion can depend on $y$ and is called appropriate for $y$ and it is so that the function $\left(y, e_{\omega}\right)_{H} \cdot e_{\omega}$ is Lebesgue integrable on $\Omega_{k}$ and

$$
y=\int_{\Omega}^{(H)}\left(y, e_{\omega}\right)_{H} e_{\omega} d \mu(\omega)=\lim _{k \rightarrow \infty}(L) \int_{\Omega_{k}}^{(H)}\left(y, e_{\omega}\right)_{H} e_{\omega} d \mu(\omega) .
$$

In this work we consider a countable-finite space $\Omega$ with a countable additive measure $\mu$. If measure $\mu$ is non-negative, the orthosimilar system $\left\{e_{\omega}\right\}_{\omega \in \Omega}$ is called nonnegative.

Definition 3. A space $\Omega$ with a measure $\mu$ is called countable-finite, if $\Omega$ is represented as a countable union of subsets $\Omega_{k} \subset \Omega$ : $\bigcup_{k \geqslant 1} \Omega_{k}=\Omega, k=1,2, \ldots$, at that, $\mu\left(\Omega_{k}\right)<\infty$ for each $k$.

In this work we employ a theorem proven in by T.P. Lukashenko in work [1].

Theorem B. Let $H$ be a separable Hilbert space over field $\mathbb{R}$ or $\mathbb{C}$, space $\Omega$ with countable additive measure $\mu$ be countable finite, $\left\{e_{\omega}\right\}_{\omega \in \Omega}$ a system of elements in $H$, and for each $y \in H$ the Parseval identity

$$
\|y\|_{H}^{2}=\int_{\Omega}\left|\left(y, e_{\omega}\right)_{H}\right|^{2} d \mu(\omega)
$$

hold true. Then $\left\{e_{\omega}\right\}_{\omega \in \Omega}$ is an orthosimilar expansion system in $H$ (in the sense of Definition 2).

We shall prove that if $H$ is a separable reproducing kernel Hilbert space over field $\mathbb{C}$ consisting of functions $f(z), z \in M$, where $M$ is a some set, then we can give an equivalent definition of orthosimilar expansion system.

Definition 4. Let $H$ be a separable reproducing kernel Hilbert space over field $\mathbb{C}$, and $\Omega$ is a space with countable additive measure $\mu$ (see [4]). A system of elements $\left\{e_{\omega}(z), z \in M\right\}_{\omega \in \Omega}$ is called orthosimilar (similar to orthogonal) expansion system with measure $\mu$ in $H$, if each function $f \in H$ is represented as

$$
f(z)=\int_{\Omega}\left(f(\tau), e_{\omega}(\tau)\right)_{H} e_{\omega}(z) d \mu(\omega), z \in M
$$

The latter identity is treated "pointwise" for each $z \in M$, and the integral is treated as the usual Lebesgue integral.

As it is noted in work [2], function $f_{\omega}=\left(f(\tau), e_{\omega}(z)\right)_{H}$ of variable $\omega \in \Omega$ is not necessary $\mu$-measurable. Because of this fact, in [2] the notion of measurable orthosimilar expansion system was introduced. 
Definition 5. Suppose that in a Hilbert space $H$ there is an orthosimilar expansion system (in the sense of Definition 2) $\left\{e_{\omega}(z)\right\}_{\omega \in \Omega}$ with a measure $\mu$. This system is called measurable if for each $f \in H$ function $f_{e}(\omega) \stackrel{\text { def }}{=}\left(f(z), e_{\omega}(z)\right)_{H}$ is $\mu$-measurable on $\Omega$.

As it was proven in work [2], for each considered orthosimilar expansion system $\left\{e_{\omega}(z)\right\}_{\omega \in \Omega}$ there exists a function $\theta(\omega),|\theta(\omega)|=1$, such that the system $\left\{\theta(\omega) \cdot e_{\omega}(z)\right\}_{\omega \in \Omega}$ is measurable.

Theorem $\mathbf{C}([2])$. If $\left\{e_{\omega}\right\} \subset H$ is a non-negative orthosimilar expansion system in $H$, and the space with measure $\Omega$ is countable-finite, then there exists a function $\theta(\omega)$ with values either in $\mathbb{R}$ or in $\mathbb{C}$ subject over which field we consider $H$ such that $|\theta(\omega)|=1$, on $\Omega$ and $\left\{\theta(\omega) \cdot e_{\omega}\right\}$ is a measurable orthosimilar expansion system in $H$.

Let $\Omega$ be a countable-finite space with a non-negative countable additive measure $\mu$. Consider the system of functions $\left\{e_{\omega}(z)\right\}_{z \in M}$ of variable $\omega \in \Omega$. Without loss of generality we can assume that this system has the property: for each $z \in M$ the function $e_{\omega}(z), \omega \in \Omega$ is $\mu$-measurable on $\Omega$. If it is not true, then there exists a complex-valued function $\theta(\omega),|\theta(\omega)|=1$ such that all the functions of the system $\left\{\theta(\omega) \cdot e_{\omega}(z)\right\}_{z \in M}$ are $\mu$-measurable (see [2]). Assume also that for each $z \in M$

$$
\int_{\Omega}\left|e_{\omega}(z)\right|^{2} d \mu(\omega)<\infty
$$

By Cauchy-Schwarz inequality each finite linear combination of the elements of system $\left\{e_{\omega}(z)\right\}_{z \in M}$ is square summable over $\Omega$ w.r.t. measure $\mu$. By $R(\Omega, \mu)$ we denote the completion of a linear span of functions $\left\{e_{\omega}(z)\right\}_{z \in M}$ w.r.t. the norm

$$
\|h\|_{R} \stackrel{\text { def }}{=} \sqrt{\int_{\Omega}|h(\omega)|^{2} d \mu(\omega)}
$$

$R(\Omega, \mu)$ is a Hilber space with the scalar product

$$
(h, g)_{R}=\int_{\Omega} h(\omega) \cdot \overline{q(\omega)} d \mu(\omega) .
$$

By Riesz-Fischer theorem, each linear continuous functional $S$ over $R(\Omega, \mu)$ is generated by some element $h$ according the rule

$$
S(f)=(f, h)_{R}, \quad f \in R(\Omega, \mu) .
$$

With each linear continuous functional generated by function $h \in R(\Omega, \mu)$ we associate the function

$$
\widehat{h}(z) \stackrel{\text { def }}{=}\left(e_{\omega}(z), h(\omega)\right)_{R}=\int_{\Omega} \overline{h(\omega)} \cdot e_{\omega}(z) d \mu(\omega), \quad z \in M .
$$

We shall call this function the transform of the functional generated by function $h \in R(\Omega, \mu)$. These functions form a Hilbert space

$$
\widehat{R}(\Omega, \mu) \stackrel{\text { def }}{=}\{\widehat{h}: h \in R(\Omega, \mu)\}
$$

with the scalar product

$$
(\widehat{h}, \widehat{q})_{\widehat{R}} \stackrel{\text { def }}{=}(q, h)_{R}, \quad\|\widehat{h}\|_{\widehat{R}}=\sqrt{(\widehat{h}, \widehat{h})_{\widehat{R}}}=\|h\|_{R}, \quad h, q \in R(\Omega, \mu) .
$$

We note that space $\widehat{R}(\Omega, \mu)$ is a Hilbert one with reproducing kernel. Indeed, each element $\widehat{h} \in \widehat{R}(\Omega, \mu)$ is represented as

$$
\widehat{h}(z)=\left(e_{\omega}(z), h(\omega)\right)_{R}, \quad z \in M .
$$

For an arbitrary $z_{0} \in M$ the estimate

$$
\left|\widehat{h}\left(z_{0}\right)\right|=\left|\left(e_{\omega}\left(z_{0}\right), h(\omega)\right)_{R}\right| \leqslant\left\|e_{\omega}\left(z_{0}\right)\right\|_{R}\|h\|_{R}=\left\|e_{\omega}\left(z_{0}\right)\right\|_{R}\|\widehat{h}\|_{\widehat{R}}
$$


holds true. Hence, for each $z_{0} \in M$ the functional $\widehat{h} \rightarrow \widehat{h}\left(z_{0}\right)$ is linear and continuous functional on space $\widehat{R}(\Omega, \mu)$, this is why space $\widehat{R}(\Omega, \mu)$ is a reproducing kernel Hilbert space.

\section{MAIN RESUlT}

Theorem 1. Suppose in a separable reproducing kernel Hilbert space $H$ over field $\mathbb{C}$ there exists a system of functions $\left\{e_{\omega}(z)\right\}_{\omega \in \Omega} \subset H$, and a space $\Omega$ with countable additive measure $\mu$ is countable-finite. Let for each $z \in M$ the function $e_{\omega}(z)$ is measurable w.r.t. variable $\omega \in \Omega$. Then the following conditions are equivalent

1. System $\left\{e_{\omega}(z)\right\}_{\omega \in \Omega} \subset H$ is an orthosimilar expansion system with measure $\mu$ in space $H$ in the sense of Definition 2, i.e., each function $f$ in $H$ is represented as

$$
f(z)=\int_{\Omega}^{(H)}\left(f(\tau), e_{\omega}(\tau)\right)_{H} e_{\omega}(z) d \mu(\omega) .
$$

Here the integral is treated as that of a function with values in a Hilbert space [4, Ch. III]. The identity of two elements is treated as that of two elements in a Hilbert space.

2. System $\left\{e_{\omega}(z)\right\}_{\omega \in \Omega} \subset H$ is an orthosimilar expansion system with measure $\mu$ in space $H$ in the sense of Definition 4, i.e., each function $f$ in space $H$ is represented as

$$
f(z)=\int_{\Omega}\left(f(\tau), e_{\omega}(\tau)\right)_{H} e_{\omega}(z) d \mu(\omega), z \in M .
$$

Identity (3) is understood "pointwise" for each fixed $z \in M$, the integral is treated as the usual Lebesgue integral.

3. The system of functions $\left\{e_{\omega}(z)\right\}_{\omega \in \Omega}$ belongs to space $H$. The reproducing kernel of space $H$ reads as

$$
K_{H}(z, \xi)=\int_{\Omega} e_{\omega}(z) \cdot \overline{e_{\omega}(\xi)} d \mu(\omega), \quad z, \xi \in M
$$

Here the integral is treated as the usual Lebesgue integral. The identity is understood "pointwise".

4. Space $H$ coincides with space $\widehat{R}(\Omega, \mu)$. Spaces $H$ and $\widehat{R}(\Omega, \mu)$ comprise the same elements and for each functions $h, r \in H$ the identity

$$
(h, r)_{H}=(h, r)_{\widehat{R}}
$$

holds true.

Let us prove that Condition 1 implies Condition 2. Suppose system $\left\{e_{\omega}(z)\right\}_{\omega \in \Omega} \subset H$ is an orthosimilar expansion system with a measure $\mu$ in a space $H$ in the sense of Definition 2 . We employ the following theorem being a particular case of a theorem proven in [4]:

Theorem D. Let $H$ be a Hilbert space and $\Omega$ be a space with a measure $\mu, S$ be a linear continuous operator mapping $H$ in another Hilbert space $Y$. If a function $f: \Omega \rightarrow H$ with the values in the Hilbert space is $\mu$-integrable in the sense of [4, Ch. III], then the function Sf $: \Omega \rightarrow Y$ is also $\mu$-integrable and

$$
\int_{\Omega} S f(\omega) d \mu(\omega)=S \int_{\Omega} f(\omega) d \mu(\omega)
$$

We take an arbitrary fixed point $z_{0}$ in set $M$ and apply this theorem with delta-functional acting from space $H$ into $\mathbb{C}$ as the operator $S$ :

$$
\delta_{z_{0}}: f \longrightarrow f\left(z_{0}\right) .
$$


We obtain the identity

$$
\begin{aligned}
f\left(z_{0}\right) & =\delta_{z_{0}} f(z)=\delta_{z_{0}} \int_{\Omega}^{(H)}\left(f(\tau), e_{\omega}(\tau)\right)_{H} e_{\omega}(z) d \mu(\omega) \\
& =\int_{\Omega}\left(f(\tau), e_{\omega}(\tau)\right)_{H} \delta_{z_{0}} e_{\omega}(z) d \mu(\omega)=\int_{\Omega}\left(f(\tau), e_{\omega}(\tau)\right)_{H} e_{\omega}\left(z_{0}\right) d \mu(\omega) .
\end{aligned}
$$

The both sides of this identity are complex numbers. The integral is treated as the usual Lebesgue integral with values in $\mathbb{C}$. Since point $z_{0} \in M$ is arbitrary, $\left\{e_{\omega}(z)\right\}_{\omega \in \Omega} \subset H$ is an orthosimilar expansion system in the sense of Definition 4. Thus, Condition 1 implies Condition 2.

Let us show that Condition 2 yields Condition 3.

It is obvious that the system of functions $\left\{e_{\omega}(z)\right\}_{\omega \in \Omega} \subset H$ belongs to space $H$. Since for a fixed $\xi \in M$ the function $K_{H}(z, \xi), z \in M$, belongs to space $H$, we can substitute this function into identity (3) to obtain

$$
\begin{aligned}
K_{H}(z, \xi) & =\int_{\Omega}\left(K_{H}(\tau, \xi), e_{\omega}(\tau)\right)_{H} e_{\omega}(z) d \mu(\omega) \\
& =\int_{\Omega} \overline{\left(e_{\omega}(\tau), K_{H}(\tau, \xi)\right)_{H}} e_{\omega}(z) d \mu(\omega)=\int_{\Omega} e_{\omega}(z) \cdot \overline{e_{\omega}(\xi)} d \mu(\omega), \quad z, \xi \in M .
\end{aligned}
$$

The identity in (5) is understood "pointwise". The integral is treated as the usual Lebesgue integral. Thus, Condition 2 yields Condition 3.

Let us show that Condition 3 implies Condition 4. We first prove that if Condition 3 holds true, then space $R(\Omega, \mu)$ defined above is a Hilbert one with reproducing kernel. In identity (4) we let $\xi=z$ and obtain

$$
K_{H}(z, z)=\int_{\Omega}\left|e_{\omega}(z)\right|^{2} d \mu(\omega)<\infty, z \in M .
$$

Thus, all the functions in the system $\left\{e_{\omega}(z)\right\}_{z \in M}$ are $\mu$-measurable and square integrable over $\Omega$ w.r.t. measure $\mu$. By Cauchy-Schwarz inequality each finite linear combination of the functions in system $\left\{e_{\omega}(z)\right\}_{z \in M}$ is also measurable and square integrable over $\Omega$ w.r.t. measure $\mu$. As it was described, space $R(\Omega, \mu)$ is the completion w.r.t. the norm

$$
\|h\|_{R}=\sqrt{\int_{\Omega}|h(\omega)|^{2} d \mu(\omega)}
$$

of linear span of functions $\left\{e_{\omega}(z)\right\}_{z \in M}$.

By Condition 3, the system of functions $\left\{e_{\omega}(z)\right\}_{\omega \in \Omega}$ belongs to space $H$. We denote by $Q$ the completion of the linear span of system $\left\{e_{\omega}(z)\right\}_{\omega \in \Omega}$ w.r.t. the norm of space $H$. Thus, $Q$ is a closed subspace of space $H$. If $g \in Q$, then $\|g\|_{Q}=\|g\|_{H}$. Since $H$ is a reproducing kernel Hilbert space, the same is true for $Q$. Indeed, if $g \in Q, z \in M$, then $g \in H$

$$
|g(z)|=\left|\left(g(\tau), K_{H}(\tau, z)\right)_{H}\right| \leqslant\left\|K_{H}(\tau, z)\right\|_{H} \cdot\|g\|_{H}=\left\|K_{H}(\tau, z)\right\|_{H} \cdot\|g\|_{Q}, \quad z \in M .
$$

This is $Q$ is a reproducing kernel Hilbert space.

The system of functions $\left\{e_{\omega}(z)\right\}_{\omega \in \Omega}$ is obviously complete in space $Q$. With each linear continuous functional on $Q$ generated by a function $g \in Q$, we associate the function

$$
\widetilde{g}(\omega)=\left(e_{\omega}(z), g(z)\right)_{Q} .
$$

The set of such functions forms a Hilbert space

$$
\widetilde{Q} \stackrel{\text { def }}{=}\{\widetilde{g}: g \in Q\}
$$


with the scalar product

$$
(\widetilde{g}, \widetilde{u})_{\widetilde{Q}} \stackrel{\text { def }}{=}(u, g)_{Q},\|\widetilde{g}\|_{\widetilde{Q}}^{2}=(\widetilde{g}, \widetilde{g})_{\widetilde{Q}}=\|g\|_{Q}^{2}, \quad g, u \in Q .
$$

Let us show that $\widetilde{Q}$ is a reproducing kernel Hilbert space. Indeed, given an arbitrary point $\omega_{0} \in \Omega$, we have the estimate

$$
\left|\widetilde{g}\left(\omega_{0}\right)\right|=\left|\left(e_{\omega_{0}}(z), g(z)\right)_{Q}\right| \leqslant\left\|e_{\omega_{0}}(z)\right\|_{Q} \cdot\|g\|_{Q}=\left\|e_{\omega_{0}}(z)\right\|_{Q} \cdot\|\widetilde{g}\|_{\widetilde{Q}}
$$

Thus, $\widetilde{Q}$ is a reproducing kernel Hilbert space. Since for each $z_{0} \in M$

$$
e_{\omega}\left(z_{0}\right)=\left(e_{\omega}(z), K_{Q}\left(z, z_{0}\right)\right)_{Q},
$$

the function $e_{\omega}\left(z_{0}\right), \omega \in \Omega$, and each finite linear combination of elements of system $\left\{e_{\omega}(z)\right\}_{z \in M}$ belongs to space $\widetilde{Q}$ as a function of $\omega \in \Omega$.

Lemma 1. Space $R(\Omega, \mu)$ coincides with space $\widetilde{Q}$ and is a reproducing kernel Hilbert space.

Proof. System of functions $\left\{e_{\omega}(z)\right\}_{z \in M}$ belongs to space $\widetilde{Q}$ and is complete in it. The same system of functions $\left\{e_{\omega}(z)\right\}_{z \in M}$ belongs to space $R(\Omega, \mu)$ and is complete in it. This is why it is sufficient to show that the norm in space $\widetilde{Q}$ is an integral one

$$
\|f\|_{\widetilde{Q}}=\sqrt{\int_{\Omega}|f(\omega)|^{2} d \mu(\omega)}, \quad f \in \widetilde{Q} .
$$

In our notations

$$
\widetilde{K_{Q}(\cdot, z)}(\omega)=\left(e_{\omega}(\eta), K(\eta, z)\right)_{Q}=e_{\omega}(z), \quad z \in M
$$

We note that for each $g \in Q$

$$
g(z)=\left(g(\eta), K_{Q}(\eta, z)\right)_{Q}=\left(\widetilde{K_{Q}(\cdot, z)}(\omega), \widetilde{g}(\omega)\right)_{\widetilde{Q}}=\left(e_{\omega}(z), \widetilde{g}(\omega)\right)_{\widetilde{Q}}, \quad z \in M .
$$

The system of reproducing kernels $\left\{K_{Q}(z, w)\right\}_{w \in M}$ is complete in space $Q$ (see [3]). Each element $f \in Q$ can be approximated in the norm of space $Q$ by finite linear combination of elements in $\left\{K_{Q}(z, w)\right\}_{w \in M}$. Namely, there exists a sequence of functions

$$
p_{n}(z) \stackrel{\text { def }}{=} \sum_{j=1}^{k_{n}} a_{j, n} K_{Q}\left(z, w_{j, n}\right), n=1,2, \ldots,
$$

where $\left\{a_{j, n}\right\}_{j, n \in \mathbb{N}}$ is a sequence of complex numbers, and $\left\{w_{j, n}\right\}_{j, n \in \mathbb{N}}$ is a sequence of points in $M$ with the property

We note that by identity (7)

$$
\left\|f(z)-p_{n}(z)\right\|_{Q} \rightarrow 0, n \rightarrow \infty .
$$

$$
\begin{aligned}
\widetilde{p}_{n}(\omega) & =\left(e_{\omega}(z), p_{n}(z)\right)_{Q}=\left(e_{\omega}(z), \sum_{j=1}^{k_{n}} a_{j, n} K_{Q}\left(z, w_{j, n}\right)\right)_{Q} \\
& =\sum_{j=1}^{k_{n}} \overline{a_{j, n}}\left(e_{\omega}(z), K_{Q}\left(z, w_{j, n}\right)\right)_{Q}=\sum_{j=1}^{k_{n}} \overline{a_{j, n}} e_{\omega}\left(w_{j, n}\right) .
\end{aligned}
$$

Thus, function $\widetilde{p}_{n}(\omega), n=1,2, \ldots$, is a finite linear combination of elements of system $\left\{e_{\omega}(z)\right\}_{z \in M}$. The obvious identity

$$
\left\|\widetilde{f}(\omega)-\widetilde{p}_{n}(\omega)\right\|_{\widetilde{Q}}=\left\|f(z)-p_{n}(z)\right\|_{Q}
$$

show that the system $\left\{e_{\omega}(z)\right\}_{z \in M}$ is complete in space $\widetilde{Q}$. We observe that identity (4) implies

$$
K_{Q}\left(z, \xi_{0}\right)=\int_{\Omega} \overline{e_{\omega}\left(\xi_{0}\right)} e_{\omega}(z) d \mu(\omega)=\int_{\Omega}\left(K_{Q}\left(\tau, \xi_{0}\right), e_{\omega}(\tau)\right)_{Q} e_{\omega}(z) d \mu(\omega) .
$$


Let us prove by induction that for each function

$$
r_{n}(z) \stackrel{\text { def }}{=} \sum_{j=1}^{n} a_{j} K_{Q}\left(z, \xi_{j}\right), \quad z \in M, \quad\left\{\xi_{j}\right\}_{j=1}^{n} \in M
$$

the identity

$$
r_{n}(z)=\int_{\Omega}\left(r_{n}(\tau), e_{\omega}(\tau)\right)_{Q} e_{\omega}(z) d \mu(\omega)
$$

holds true. For $n=1$ it follows from (11) and the linearity of scalar product. Suppose that identity (12) holds for $n=n_{0}$. Let us show that identity 12 is valid for $n=n_{0}+1$. It is easy to see that

Then the identity

$$
r_{n_{0}+1}(z)=r_{n_{0}}(z)+a_{n_{0}+1} K_{Q}\left(z, \xi_{n_{0}+1}\right) .
$$

$$
\begin{aligned}
r_{n_{0}+1}(z) & =r_{n_{0}}(z)+a_{n_{0}+1} K_{Q}\left(z, \xi_{n_{0}+1}\right) \\
& =\int_{\Omega}\left(r_{n_{0}}(\tau), e_{\omega}(\tau)\right)_{Q} e_{\omega}(z) d \mu(\omega)+a_{n_{0}+1} \int_{\Omega}\left(K_{Q}\left(\tau, \xi_{n_{0}+1}\right), e_{\omega}(\tau)\right)_{Q} e_{\omega}(z) d \mu(\omega) \\
& =\int_{\Omega}\left(r_{n_{0}}(\tau), e_{\omega}(\tau)\right)_{Q} e_{\omega}(z) d \mu(\omega)+\int_{\Omega}\left(a_{n_{0}+1} K_{Q}\left(\tau, \xi_{n_{0}+1}\right), e_{\omega}(\tau)\right)_{H} e_{\omega}(z) d \mu(\omega) \\
& =\int_{\Omega}\left(r_{n_{0}}(\tau)+a_{n_{0}+1} K_{Q}\left(\tau, \xi_{n_{0}+1}\right), e_{\omega}(\tau)\right)_{Q} e_{\omega}(z) d \mu(\omega) \\
& =\int_{\Omega}\left(r_{n_{0}+1}(\tau), e_{\omega}(\tau)\right)_{Q} e_{\omega}(z) d \mu(\omega)
\end{aligned}
$$

holds true. Thus, we have proven that identity (12) is valid, and thus for each function $p_{n}(z)$ (see (8)) the representation

$$
p_{n}(z)=\int_{\Omega}\left(p_{n}(\tau), e_{\omega}(\tau)\right)_{Q} e_{\omega}(z) d \mu(\omega)
$$

is true. It follows from identity (13) that for each $\xi_{0} \in M$ we have

$$
\left(p_{n}(\eta), K_{Q}\left(\eta, \xi_{0}\right)\right)_{Q}=\int_{\Omega}\left(p_{n}(\tau), e_{\omega}(\tau)\right)_{Q}\left(e_{\omega}(\eta), K_{Q}\left(\eta, \xi_{0}\right)\right)_{Q} d \mu(\omega)
$$

Since function $p_{n}(z)$ is a finite linear combination of the elements in system $\left\{K_{Q}(z, \xi)\right\}_{\xi \in M}$, then by employing (15) and the linearity of integral and scalar product one can prove easily that

$$
\left\|p_{n}\right\|_{Q}^{2}=\left(p_{n}(z), p_{n}(z)\right)_{Q}=\int_{\Omega}\left(p_{n}(z), e_{\omega}(z)\right)_{Q}\left(e_{\omega}(z), p_{n}(z)\right)_{Q} d \mu(\omega) .
$$

As it was mentioned above, see (10),

$$
\widetilde{p}_{n}(\omega)=\left(e_{\omega}(z), p_{n}(z)\right)_{Q}
$$

At that, $\left\|\widetilde{p}_{n}\right\|_{\widetilde{Q}}=\left\|p_{n}\right\|_{Q}$. This is why it follows from (16) that

$$
\begin{aligned}
\left\|\widetilde{p}_{n}\right\|_{\widetilde{Q}}^{2} & =\left\|p_{n}\right\|_{Q}^{2}=\int_{\Omega}\left(p_{n}(z), e_{\omega}(z)\right)_{Q}\left(e_{\omega}(z), p_{n}(z)\right)_{Q} d \mu(\omega) \\
& =\int_{\Omega} \widetilde{p}_{n}(\omega) \cdot{\widetilde{p_{n}}}_{(\omega)} d \mu(\omega)=\int_{\Omega}\left|\widetilde{p}_{n}(\omega)\right|^{2} d \mu(\omega) .
\end{aligned}
$$

We shall make use of Fatou theorem (see, for instance, [6]).

Theorem E. If a sequence of measurable nonnegative functions $\left\{y_{n}\right\}$ converges a.e. in $\Omega$ to a function $y$ and

$$
\int_{\Omega} y_{n}(\omega) d \mu(\omega) \leqslant K
$$


where $K$ is a constant, then $y$ is integrable over $\Omega$ and

$$
\int_{\Omega} y(\omega) d \mu(\omega) \leqslant K
$$

We let $y_{n}(\omega)=\left|\widetilde{p}_{n}(\omega)\right|^{2}$. The sequence $\left\{\left|\widetilde{p}_{n}(\omega)\right|^{2}\right\}_{n \geqslant 0}$ converges pointwise in $\Omega$ to the function $y(\omega)=|\widetilde{f}(\omega)|^{2}$. Indeed, the sequence of function $\left\{p_{n}\right\}_{n \geqslant 0}$ converges to function $f$ in the norm of space $Q$ (see $(9)$ ), and thus for each $\omega_{0} \in \Omega$

$$
\begin{aligned}
|| \widetilde{p}_{n}\left(\omega_{0}\right)|-| \widetilde{f}\left(\omega_{0}\right)|| & \leqslant\left|\widetilde{p}_{n}\left(\omega_{0}\right)-\widetilde{f}\left(\omega_{0}\right)\right|=\left|\left(e_{\omega_{0}}(z), p_{n}(z)-f(z)\right)_{Q}\right| \\
& \leqslant\left\|e_{\omega_{0}}(z)\right\|_{Q} \cdot\left\|p_{n}(z)-f(z)\right\|_{Q} \longrightarrow 0, \quad n \rightarrow \infty .
\end{aligned}
$$

The function $u=x^{2}, x \geqslant 0$, is continuous and thus it follows from (18) that

$$
\left.|| \widetilde{p}_{n}\left(\omega_{0}\right)\right|^{2}-\left|\widetilde{f}\left(\omega_{0}\right)\right|^{2} \mid \longrightarrow 0, \quad n \rightarrow \infty .
$$

Since

$$
\left\|\widetilde{p}_{n}\right\|_{\widetilde{Q}}^{2} \rightarrow\|\widetilde{f}\|_{\widetilde{Q}}^{2}, \quad n \rightarrow \infty
$$

there exists a number $\varepsilon>0$ such that

$$
\int_{\Omega}\left|\widetilde{p}_{n}(\omega)\right|^{2} d \mu(\omega)=\left\|\widetilde{p}_{n}\right\|_{\widetilde{Q}}^{2} \leqslant\|\widetilde{f}\|_{\widetilde{Q}}^{2}+\varepsilon, \quad n=1,2, \ldots
$$

By Fatou theorem the function $|\widetilde{f}(\omega)|^{2}$ is integrable over $\Omega$ w.r.t. measure $\mu$ and the inequality

$$
\int_{\Omega}|\widetilde{f}(\omega)|^{2} d \mu(\omega) \leqslant\|\widetilde{f}\|_{\widetilde{Q}}^{2}+\varepsilon
$$

holds true. We consider the sequence $\left\{p_{n}\right\}_{n=N}^{\infty}$, where $N$ is a natural number. Due to (19), increasing $N$, we can make the number $\varepsilon>0$ as small as needed. In inequality (20) the left hand side is independent of $\varepsilon$. Thus,

$$
\int_{\Omega}|\widetilde{f}(\omega)|^{2} d \mu(\omega) \leqslant\|\widetilde{f}\|_{\widetilde{Q}}^{2}, \quad \widetilde{f} \in \widetilde{Q} .
$$

Let us prove that

$$
\int_{\Omega}|\widetilde{f}(\omega)|^{2} d \mu(\omega)=\|\widetilde{f}\|_{\widetilde{Q}}^{2}, \quad \widetilde{f} \in \widetilde{Q}
$$

We consider two functions

$$
\begin{gathered}
u: \widetilde{Q} \longrightarrow \mathbb{R}, \quad u(\widetilde{f})=\|\widetilde{f}\|_{\widetilde{Q}} \\
v: \widetilde{Q} \longrightarrow \mathbb{R}, \quad v(\widetilde{f})=\sqrt{\int_{\Omega}|\widetilde{f}(\omega)|^{2} d \mu(\omega)} .
\end{gathered}
$$

By the triangle inequality we get

$$
u(\widetilde{f}) \leqslant u(\widetilde{f}-\widetilde{g})+u(\widetilde{g}), \quad \widetilde{f}, \widetilde{g} \in \widetilde{Q}
$$

that implies

$$
|u(\widetilde{f})-u(\widetilde{g})| \leqslant u(\widetilde{f}-\widetilde{g}), \quad \widetilde{f}, \widetilde{g} \in Q
$$

Hence, the function $u: \widetilde{Q} \longrightarrow \mathbb{R}$ is continuous. By inequality 21 function $v$ is defined on $\widetilde{Q}$. Cauchy-Schwarz inequality yields

$$
v(\widetilde{f}) \leqslant v(\widetilde{f}-\widetilde{g})+v(\widetilde{g}), \quad \widetilde{f}, \widetilde{g} \in \widetilde{Q},
$$

and thus, employing (21), we obtain

$$
|v(\widetilde{f})-v(\widetilde{g})| \leqslant v(\widetilde{f}-\widetilde{g}) \leqslant u(\widetilde{f}-\widetilde{g}), \quad \widetilde{f}, \widetilde{g} \in \widetilde{Q} .
$$


Hence, the function $v: \widetilde{Q} \longrightarrow \mathbb{R}$ is continuous. Identity 17 means that everywhere on a dense set $\widetilde{Q}$ being the linear span of the system $\left\{e_{\omega}(z)\right\}_{z \in M}$, continuous functions $u$ and $v$ coincide. If the sequence $\left\{\widetilde{p}_{n}\right\}_{n \geqslant 0}$ of finite linear combination of the system $\left\{e_{\omega}(z)\right\}_{z \in M}$ approximates an element $\widetilde{f} \in \widetilde{Q}$, then

$$
u\left(\widetilde{p}_{n}\right)=v\left(\widetilde{p}_{n}\right), \quad n=1,2, \ldots
$$

Employing the continuity of functions $u$ and $v$, we obtain

$$
u(\widetilde{f})=v(\widetilde{f}), \quad \widetilde{f} \in \widetilde{Q} .
$$

Thus, for each $\widetilde{f} \in \widetilde{Q}$ the identity $(22)$ holds true.

As it was mentioned above, functions $\widetilde{p}_{n}(\omega), n=1,2, \ldots$, are finite linear combinations of the elements in system $\left\{e_{\omega}(z)\right\}_{z \in M}$. We note now that space $\widetilde{Q}$ can be considered as the completion of the linear span of the system $\left\{e_{\omega}(z)\right\}_{z \in M}$ w.r.t. the norm $\|\cdot\|_{\widetilde{Q}}$. As it was mentioned above, space $R(\Omega, \mu)$ is the completion of the linear span of the system $\left\{e_{\omega}(z)\right\}_{z \in M}$ w.r.t. the norm

$$
\|h\|_{R(\Omega, \mu)} \stackrel{\text { def }}{=} \sqrt{\int_{\Omega}|h(\omega)|^{2} d \mu(\omega)} .
$$

This is why spaces $\widetilde{Q}$ and $R(\Omega, \mu)$ coincide. Therefore, space $R(\Omega, \mu)$ is one with reproducing kernel. The proof of Lemma 1 is complete.

The next theorem holds true.

Theorem 2. Let $H$ be a separable reproducing kernel Hilbert space consisting of the functions defined on a countable-finite space $\Omega$ with a countable-additive measure $\mu$. The norm of space $H$ has the integral form

$$
\|f\|_{H}=\sqrt{\int_{\Omega}|f(\xi)|^{2} d \mu(\xi)}
$$

if and only if the system of the functions $\left\{K_{H}(\xi, t)\right\}_{t \in \Omega}$ is an orthosimilar expansion system with measure $\mu$ in space $H$ in the sense of Definition 2.

Proof. Necessity. Let a system of functions $\left\{K_{H}(\xi, t)\right\}_{t \in \Omega}$ is the orthosimilar expansion system with measure $\mu$ in space $H$ in the sense of Definition 2. It means that each function $f \in H$ is represented as

$$
f(z)=\int_{\Omega}^{(H)}\left(f(\tau), K_{H}(\tau, \xi)\right)_{H} K_{H}(z, \xi) d \mu(\xi) .
$$

Then an analogue of Parseval identityy for orthosimilar expansion systems holds true [1, Thm. 1]), i.e., for each $f \in H$ we have

$$
\|f\|_{H}^{2}=\int_{\Omega}\left|\left(f(\tau), K_{H}(\tau, \xi)\right)_{H}\right|^{2} d \mu(\xi)=\int_{\Omega}|f(\xi)|^{2} d \mu(\xi) .
$$

Thus, the identity (25) is valid. The necessity is proven.

Sufficiency. Suppose that the norm in space $H$ reads as (25). It means that

$$
\|f\|_{H}^{2}=\int_{\Omega}|f(\xi)|^{2} d \mu(\xi)=\int_{\Omega}\left|\left(f(\tau), K_{H}(\tau, \xi)\right)_{H}\right|^{2} d \mu(\xi) .
$$

Thus, for system of functions $\left\{K_{H}(\xi, t)\right\}_{t \in \Omega}$ the analogue of Parseval identity holds true [1]. By Theorem B, system of functions $\left\{K_{H}(\xi, t)\right\}_{t \in \Omega}$ is an orthosimilar expansion system in the sense of Definition 2. The proof is complete. 
The norm in space $R(\Omega, \mu)$ has the integral form and since $R(\Omega, \mu)$ is a reproducing kernel Hilbert space, by Theorem 2 , the system of reproducing kernels $\left\{K_{R}(\omega, t)\right\}_{t \in \Omega}$ of space $R(\Omega, \mu)$ is an orthosimilar expansion system in space $R(\Omega, \mu)$ in the sense of Definition 2. As we have already proven, it implies that system $\left\{K_{R}(\omega, t)\right\}_{t \in \Omega}$ is an orthosimilar expansion system in the sense of Definition 4.

Lemma 2. Suppose we are given a space $\Omega$ with a countable additive measure $\mu$ and let in a separable Hilbert space $H$ consisting of functions defined on space $\Omega$ the system of reproducing kernels $\left\{K_{H}(z, \xi)\right\}_{\xi \in \Omega}$ is an orthosimilar expansion system in the sense of Definition 4, i.e., each element $f$ in space $H$ can be represented as

$$
f(z)=\int_{\Omega}\left(f(\tau), K_{H}(\tau, \xi)\right)_{H} K_{H}(z, \xi) d \mu(\xi), \quad z \in \Omega .
$$

Then system $\left\{K_{H}(z, \xi)\right\}_{\xi \in \Omega}$ is an orthosimilar expansion system in the sense of Definition 2, i.e., each $f$ in space $H$ is represented as

$$
f(z)=\int_{\Omega}^{(H)}\left(f(\tau), K_{H}(\tau, \xi)\right)_{H} K_{H}(z, \xi) d \mu(\xi) .
$$

Proof. The system of reproducing kernels $\left\{K_{H}(z, \xi)\right\}_{\xi \in \Omega}$ is complete in space $H$ [3]. As in the proof of Lemma 1, one can show that if $\left\{p_{n}(z)\right\}_{n \geqslant 0}$ is a sequence of finite linear combinations of the elements of system $\left\{K_{H}(z, \xi)\right\}_{\xi \in \Omega}$ approximating an element $f \in H$, then

$$
\left\|p_{n}\right\|_{H}^{2}=\int_{\Omega}\left(p_{n}(\tau), K_{H}(\tau, \xi)\right)_{H}\left(K_{H}(\tau, \xi), p_{n}(\tau)\right)_{H} d \mu(\xi)=\int_{\Omega}\left|p_{n}(\xi)\right|^{2} d \mu(\xi), \quad n=1,2, \ldots
$$

We let $y_{n}(\xi)=\left|p_{n}(\xi)\right|^{2}$. The sequence of functions $\left\{\left|p_{n}(\xi)\right|^{2}\right\}_{n \geqslant 0}$ converges pointwise in $\Omega$ to the function $y(\xi)=|f(\xi)|^{2}$, and

$$
\int_{\Omega}\left|p_{n}(\xi)\right|^{2} d \mu(\xi)=\left\|p_{n}\right\|_{H}^{2} \leqslant\|f\|_{H}^{2}+\varepsilon, \quad n=1,2, \ldots,
$$

where $\varepsilon$ is a positive number independent of $n$. By Fatou theorem, function $f(\xi)$ is integrable over $\Omega$ w.r.t. measure $\mu$ and the inequality

$$
\int_{\Omega}|f(\xi)|^{2} d \mu(\xi) \leqslant\|f\|_{H}^{2}+\varepsilon
$$

holds true. Considering the sequence $\left\{p_{n}\right\}_{n \geqslant N}$ for sufficiently large $N$, we can make $\varepsilon$ as small as needed. The left hand side in inequality (27) is independent of $\varepsilon$. This is why

$$
\int_{\Omega}|f(\xi)|^{2} d \mu(\xi) \leqslant\|f\|_{H}^{2}, \quad f \in H
$$

Let us prove that

We consider two functions

$$
\int_{\Omega}|f(\xi)|^{2} d \mu(\xi)=\|f\|_{H}^{2}, \quad f \in H .
$$

$$
\begin{gathered}
u: H \longrightarrow \mathbb{R}, \quad u(f)=\|f\|_{H}, \\
v: H \longrightarrow \mathbb{R}, \quad v(f)=\sqrt{\int_{\Omega}|f(\xi)|^{2} d \mu(\xi) .}
\end{gathered}
$$

By the triangle inequality we have

$$
u(f) \leqslant u(f-g)+u(g), \quad f, g \in H .
$$

It implies

$$
|u(f)-u(g)| \leqslant u(f-g), \quad f, g \in H
$$


Hence, the function $u: H \longrightarrow \mathbb{R}$ is continuous. By inequality (28), function $v$ is defined on $H$. By Cauchy-Schwarz inequality

$$
v(f) \leqslant v(f-g)+v(g), \quad f, g \in H,
$$

and employing (28), we obtain

$$
|v(f)-v(g)| \leqslant v(f-g) \leqslant u(f-g), \quad f, g \in H .
$$

Thus, the function $v: H \longrightarrow \mathbb{R}$ is continuous. Identity (26) means that continuous functions $u$ and $v$ coincide on a dense subset of $H$, which is the linear span of system $\left\{K_{H}(z, \xi)\right\}_{\xi \in \Omega}$. If a sequence $p_{n}$ of finite linear combinations of the elements in system $\left\{K_{H}(z, \xi)\right\}_{\xi \in \Omega}$ approximates an element $f \in H$, then

$$
u\left(p_{n}\right)=v\left(p_{n}\right), \quad n=1,2, \ldots,
$$

and in view of the continuity of functions $u$ and $v$, we obtain

$$
u(f)=v(f), \quad f \in H .
$$

Hence, for each $f \in H$ identity (29) holds true. Identity 29) means that the analogue of Parseval identity for system $\left\{K_{H}(z, \xi)\right\}_{\xi \in \Omega}$ holds:

$$
\|f\|_{H}^{2}=\int_{\Omega}|f(\xi)|^{2} d \mu(\xi)=\int_{\Omega}\left|\left(f(\tau), K_{H}(\tau, \xi)\right)_{H}\right|^{2} d \mu(\xi), f \in H .
$$

Since measure $\mu$ is countable additive, by Theorem B the latter identity implies that the system of reproducing kernles is orthosimilar in the sense of Definition 2, i.e., each element is represented as

The proof is complete.

$$
f(z)=\int_{\Omega}^{(H)}\left(f(\tau), K_{H}(\tau, \xi)\right)_{H} K_{H}(z, \xi) d \mu(\xi) .
$$

Consider the space

$$
\overline{R(\Omega, \mu)} \stackrel{\text { def }}{=}\left\{\bar{h}: h \in R(\Omega, \mu),(\bar{h}, \bar{r})_{\bar{R}} \stackrel{\text { def }}{=}(r, h)_{R}\right\} .
$$

Each function $h \in R(\Omega, \mu)$ can be represented as

$$
h(\omega)=\int_{\Omega}^{(R)}\left(h(\tau), K_{R}(\tau, t)\right)_{H} K_{R}(\omega, t) d \mu(t) .
$$

It yields

$$
h(\omega)=\int_{\Omega}\left(h(\tau), K_{R}(\tau, t)\right)_{H} K_{R}(\omega, t) d \mu(t), \quad \omega \in \Omega .
$$

We take the complex conjugation of this identity to obtain

$$
\overline{h(\omega)}=\int_{\Omega} \overline{\left(h(\tau), K_{R}(\tau, t)\right)_{R} K_{R}(\omega, t)} d \mu(t)=\int_{\Omega}\left(\overline{h(\tau)}, \overline{K_{R}(\tau, t)}\right)_{\bar{R}} \overline{K_{R}(\omega, t)} d \mu(t), \quad \omega \in \Omega .
$$

Due to Lemma 2

$$
\overline{h(\omega)}=\int_{\Omega}^{(\bar{R})}\left(\overline{h(\tau)}, \overline{K_{R}(\tau, t)}\right)_{\bar{R}} \overline{K_{R}(\omega, t)} d \mu(t) .
$$

Identity 35 means that the system of functions $\left\{\overline{K_{R}(\omega, t)}\right\}_{t \in \Omega}$ is an orthosimilar expansion system in the sense of Definition 2 in space $\overline{R(\Omega, \mu)}$.

The operator $\mathbb{T}$ acting from $\overline{R(\Omega, \mu)}$ into $\widehat{R}(\Omega, \mu)$ by the rule

$$
\mathbb{T}: \bar{h} \longrightarrow \widehat{h}(z) \stackrel{\text { def }}{=} \int_{\Omega} \overline{h(\omega)} \cdot e_{\omega}(z) d \mu(\omega), \quad z \in M,
$$


is a linear continuous operator (the definition of space $\widehat{R}(\Omega, \mu)$ was given above). We apply operator $\mathbb{T}$ to identity (35) and employ Theorem $\mathrm{C}$ to get

$$
\begin{aligned}
\widehat{h}(z) & =\mathbb{T} \int_{\Omega}^{(\bar{R})}\left(\overline{h(\tau)}, \overline{K_{R}(\tau, t)}\right)_{\bar{R}} \overline{K_{R}(\omega, t)} d \mu(t)=\int_{\Omega}^{(\widehat{R})}\left(\overline{h(\tau)}, \overline{K_{R}(\tau, t)}\right)_{\bar{R}} \mathbb{T} \overline{K_{R}(\omega, t)} d \mu(t) \\
& =\int_{\Omega}^{(\widehat{R})}\left(\overline{h(\tau)}, \overline{K_{R}(\tau, t)}\right)_{\bar{R}} \widehat{K}_{R}(z, t) d \mu(t)=\int_{\Omega}^{(\widehat{R})}\left(\overline{h(\tau)}, \overline{K_{R}(\tau, t)}\right)_{\bar{R}} \cdot e_{t}(z) d \mu(t) .
\end{aligned}
$$

In the latter identity we have employed the fact that

$$
\widehat{K}_{R}(z, t)=\int_{\Omega} \overline{K_{R}(\omega, t)} \cdot e_{\omega}(z) d \mu(\omega)=e_{t}(z), z \in M
$$

As it was noticed above (see the definition of space $R(\Omega, \mu)$ )

$$
\left(\overline{h(\tau)}, \overline{K_{R}(\tau, t)}\right)_{\bar{R}}=\overline{\left(h(\tau), K_{R}(\tau, t)\right)_{R}}=\left(K_{R}(\tau, t), h(\tau)\right)_{R}=\left(\widehat{h}(z), e_{t}(z)\right)_{\widehat{R}} .
$$

Substituting relation (37) into identity (36), we obtain

$$
\widehat{h}(z)=\int_{\Omega}^{(\widehat{R})}\left(\widehat{h}(z), e_{t}(z)\right)_{\widehat{R}} \cdot e_{t}(z) d \mu(t) .
$$

The latter means that the system of functions $\left\{e_{\omega}(z)\right\}_{\omega \in \Omega}$ is an orthosimilar expansion system in the sense of Definition 2 in space $\widehat{R}(\Omega, \mu)$. As it was noted above, space $\widehat{R}(\Omega, \mu)$ is a reproducing kernel Hilbert space.

Let us calculate the reproducing kernel of space $\widehat{R}(\Omega, \mu)$.

In order to do it, we substitute an element $K_{\widehat{R}}(z, \xi)$ for fixed $\xi \in M$ as $\widehat{h}$ into identity 38. Then one can show easily that the reproducing kernel in space $\widehat{R}(\Omega, \mu)$ reads as

$$
K_{\widehat{R}}(z, \xi)=\int_{\Omega} e_{\omega}(z) \cdot \overline{e_{\omega}(\xi)} d \mu(\omega), \quad z, \xi \in M
$$

On the other hand, identity (4) holds true

$$
K_{H}(z, \xi)=\int_{\Omega} e_{t}(z) \cdot \overline{e_{t}(\xi)} d \mu(t), \quad z, \xi \in M .
$$

By Moore-Aronszajn theorem, space $H$ coincides with space $\widehat{R}(\Omega, \mu)$, i.e., these spaces consist of the same elements and the identity

$$
(f, g)_{H}=(f, g)_{\widehat{R}}, \quad f, g \in H
$$

holds true. Thus, Condition 3 implies Condition 4 of Theorem 1.

Suppose Condition 4 of Theorem 1, i.e., space $H$ coincides with space $\widehat{R}(\Omega, \mu)$. By construction, system $\left\{e_{\omega}(z)\right\}_{\omega \in \Omega}$ is an orthosimilar expansion system in the sense of Definition 2 space $\widehat{R}(\Omega, \mu)$. It means that in space $H$ system $\left\{e_{\omega}(z)\right\}_{\omega \in \Omega}$ is an orthosimilar expansion system in the sense of Definition 2, i.e. Condition 1 holds true. Theorem is proven.

\section{EXAMPLES}

2.1. Weighted Hilbert transform in Bergman space. Let $G$ be a simply connected Jordan domain in $\mathbb{C}$. As system $\left\{e_{\omega}(z)\right\}_{\omega \in \Omega}$ we take functions $\left\{\frac{1}{(z-\xi)^{2}}\right\}_{\xi \in G}$ defined on the set $M=\mathbb{C} \backslash \bar{G}$. As $\Omega$ we take domain $G$; in domain $G$ we have a countable finite measure $\mu$. We choose measure $\mu$ so that the space

$$
B_{2}(G, \mu) \stackrel{\text { def }}{=}\left\{f \in H(G):\|f\|_{B_{2}}^{2}=\int_{G}|f(z)|^{2} d \mu(z)<\infty\right\}
$$


consisting of analytic and square integrable w.r.t. measure $\mu$ in domain $G$ functions is a separable reproducing kernel Hilbert space and the system of functions $\left\{\frac{1}{(z-\xi)^{2}}\right\}_{\xi \in G}$ is complete in this space. Space $\widetilde{B}_{2}(G, \mu)$ is defined as the set of functions

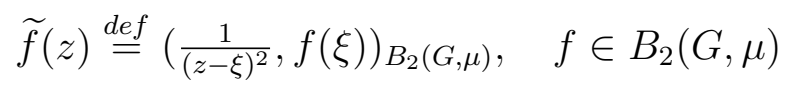

with the scalar product

$$
(\widetilde{f}, \widetilde{g})_{\widetilde{B}_{2}(G, \mu)} \stackrel{\text { def }}{=}(g, f)_{B_{2}(G, \mu)}, \quad \widetilde{g}, \widetilde{f} \in \widetilde{B}_{2}(G, \mu) .
$$

Under these assumptions Theorem 1 holds true.

As space $R(\Omega, \mu)$ we take space $B_{2}(G, \mu)$, and as $\widehat{R}(\Omega, \mu)$ we take space $\widetilde{B}_{2}(G, \mu)$. The orthosimilar system $\left\{\frac{1}{(z-\xi)^{2}}\right\}_{\xi \in G}$ and the problem on description of the dual space for $B_{2}(G, \mu)$ were considered in [7].

2.2. Weighted Fourier-Laplace transform in Bergman space. As $\Omega$ we take here a convex domain $G$ in the complex plane with some measure $\mu$ satisfying the hypothesis of Theorem 1. As system $\left\{e_{\omega}(z)\right\}_{\omega \in \Omega}$, we take the system of functions $\left\{e^{\xi z}\right\}_{\xi \in G}, M=\mathbb{C}$, and as space $R(\Omega, \mu)$ we take space $B_{2}(G, \mu)$. As space $\widehat{R}(\Omega, \mu)$ we choose the space $\widehat{B}_{2}(G, \mu)$ consisting of functions

$$
\widehat{f}(z)=\left(e^{z \cdot \xi}, f(\xi)\right)_{B_{2}}=\int_{G} \overline{f(\xi)} \cdot e^{z \cdot \xi} d \mu(\xi), z \in \mathbb{C}, \quad f \in B_{2}(G, \mu)
$$

At that,

$$
(\widehat{f}, \widehat{h})_{\widehat{B}_{2}} \stackrel{\text { def }}{=}(h, f)_{B_{2}}, \quad \widehat{h}, \widehat{f} \in \widehat{B}_{2}(G, \mu) .
$$

Then Theorem 1 holds true.

\section{BIBLIOGRAPHY}

1. T.P. Lukashenko. Properties of expansion systems similar to orthogonal ones // Izv. RAN. Ser. Matem. 1998. V. 62, No. 5. P. 187-206. [Izv. Math. 1998. V. 62, No. 5. P. 1035-1054.]

2. T.P. Lukashenko. Coefficients with respect to orthogonal-like decomposition systems // Matem. Sbornik. 1997. V. 188, No. 12. P. 57-72. [Sb. Math. 1997. V. 188, No. 12. P. 1783-1798.]

3. N. Aronszajn. Theory of reproducing kernels // Trans. Amer. Math. Soc. 1990. V. 68, No. 3. P. 337-404.

4. N. Dunford, J.T. Schwartz. Linear operators. Part I. General theory. Wiley Interscience, New York, 1958.

5. H. Hedenmalm, B. Korenblum, K. Zhu. Theory of Bergman spaces. Springer-Verlag, New York, 2000.

6. A.N. Kolmogorov, S.V. Fomin. Elements of the theory of functions and functional analysis. Nauka, Moscow, 1976. [Dover Publication, New York, 1999.]

7. V.V. Napalkov (Jr.) On an orhosimilar system in the space of analytical function and a problem of describing the dual space // Ufimskij Matem. Zhurn. 2011. V. 3, No. 1. P. 31-42. [Ufa Math. J. 2011. V. 3, No. 1. P. 31-41.]

Valerii Valentinovich Napalkov, Institute of Mathematics USC RAS,

Chernyshevskogo str., 112,

450008, Ufa, Russia

E-mail: vnap@mail.ru 